\title{
Association of Cancer Caregiver Stress and Negative Attribution Style With Depressive Symptoms and Cortisol: A Cross-Sectional Study
}

Patrick Pössel ( $\square$ patrick.possel@louisville.edu )

University of Louisville https://orcid.org/0000-0002-5851-4253

Amanda M Mitchell

University of Louisville

Brooks Harbison

University of Louisville

G. Rafael Fernandez-Botran

University of Louisville

\section{Research Article}

Keywords: informal cancer caregiving stress, depressive symptoms, negative attribution style, salivary cortisol

Posted Date: November 10th, 2021

DOI: https://doi.org/10.21203/rs.3.rs-1054279/v1

License: (9) This work is licensed under a Creative Commons Attribution 4.0 International License. Read Full License

Version of Record: A version of this preprint was published at Supportive Care in Cancer on February 18th, 2022. See the published version at https://doi.org/10.1007/s00520-022-06866-1. 


\section{Abstract}

Purpose. We examined the effect of cancer caregiver stress and negative attribution style (NAS) on depressive symptoms and salivary cortisol.

Method. The sample came from a hospital bone marrow unit and caregiver support organizations and included 60 informal cancer caregivers (51.7\% partners) of cancer patients (provided care for a median of 27.5 hours per week for 12 months) and 46 non-caregiver participants. In this cross-sectional study, participants completed questionnaires assessing NAS and depressive symptoms and provided saliva samples to measure cortisol.

Results. Linear regressions demonstrated that cancer caregiver stress $(p=0.001)$ and the cancer caregiver stress by NAS interaction ( $p=0.017)$, but not NAS alone ( $p=0.152)$, predicted depressive symptoms. Caregivers independent of their NAS and non-caregivers high in NAS reported high depression while noncaregivers low in NAS reported low depression. Neither cancer caregiver stress $(p=0.920)$ nor NAS alone $(p=0.114)$, but their interaction, predicted cortisol $(p=0.036)$. Higher NAS was associated with a higher cortisol in both groups while non-caregivers had higher cortisol than caregivers.

Conclusions. If the findings can be replicated, the implementation of interventions to support informal caregivers in managing their chronic stress and modify their NAS appears warranted.

\section{Introduction}

Cancer is one of the leading reasons for informal caregiving [1], the process by which family members and friends provide support to someone with cancer. In 2015, about 2.8 million family members and friends provided care for cancer patients in the United States [2]. Informal cancer caregiving can be a significant time commitment, with an average caregiver providing care for 30 hours a week over multiple years [3]. Given the chronicity of this emotional demanding experience, it is unsurprising that cancer caregivers report psychological stress (for a review see 4) and stress-related issues with psychological functioning including depression [5, 6]. In fact, informal cancer caregivers experience high prevalence of depression and depressive symptoms ranging from $10 \%$ to $53 \%$ (for a review see 7 ).

Psychological stress among informal caregivers is also expressed in physiological functioning such as elevated levels of cortisol (for a review see 8). Elevated cortisol levels are critical because cortisol is also associated with depression (for a meta-analysis see 9) and they are a risk factor for physical health issues like cardiovascular disease (CVD; 10,11). Regardless, the use of measures of physiological functioning in cancer caregiver research is underdeveloped (for a review see 12). This constitutes a problem as the inclusion of measures of physiological functioning into research on informal caregivers would provide a more comprehensive view of their health [13]. Studying associations among informal caregiver stress with both psychological and physiological functioning would be a first step in developing interventions to improve psychological and physical health in informal cancer caregivers. Given the clear impact of caregiver stress on psychological health, identifying modifiable factors relevant to the 
psychological and physiological health of cancer caregivers is critical. Maladaptive thought patterns have been found to contribute to depressive symptoms in cancer caregivers [14] and dementia caregivers [15]. Studies assessing the role of modifiable factors on both psychological and physiological health outcomes among cancer caregivers are warranted.

Causal attributions of a stressful situation can be modified in psychotherapy and are the mechanism underlying the effects of cognitive behavioral therapy on depressive symptoms with different populations [16]. Based on the reformulated helplessness theory [17], individuals attribute a stressful situation to a cause that is either global or specific, internal or external, and stable or unstable. When individuals have the tendency to attribute stressful situations to a global, internal, and stable cause, they demonstrate a negative attribution style (NAS). Empirical studies provide evidence for the impact of a NAS on depressive symptoms (for a review see 18). Further, prior work has described associations of NAS with measures of physical health ranging from self-reported hypertension [19] and general health [20], to specific measures of physical functioning, like T4/T8 ratio and response to stimulation by PHA [21], to incidents of CVD (22), and death caused by a coronary event $[22,23]$. However, while NAS is associated with depression (for a review see 18) and CVD [22], and cortisol is associated with depression (for a meta-analysis see 9) and CVD $[10,11]$, no previous study examined the relation between informal cancer caregiver stress and NAS with depressive symptoms and cortisol. Considering the relevance of depression (for a review see 7) and cortisol (for a review see 8) and the importance of a modifiable factor like NAS [16] for interventions to improve health in this population, this seems a crucial gap in the literature.

In the current study, we studied the effect of informal cancer caregiving and NAS on depressive symptoms and cortisol levels in saliva. Based on the reformulated helplessness theory [17] and empirical research, we predicted that (a) informal cancer caregivers will show elevated levels of depressive symptoms [6, 7] and salivary cortisol levels (for a review see 8); (b) NAS is positively related to depressive symptoms (for a review see 18) and cortisol in both caregivers and non-caregivers; (c) NAS serves as moderator [17] by increasing the effect of informal cancer caregiver stress on depressive symptoms and salivary cortisol. In particular, we expected NAS would be related to more self-reported depressive symptoms and higher cortisol levels in informal cancer caregivers.

\section{Methods}

\section{Participants}

The informal caregiver group consisted of 60 caregivers of individuals with cancer. Recruitment efforts included in-person requests at the Bone Marrow Unit of a local hospital $(62.9 \%, n=44)$, email invitations to a local cancer support organization $(20 \%, n=14)$ and members of a caregiver support group at a local cancer center $(12.9 \%, n=9)$, and listserv announcements to a university community $(4.3 \%, n=3)$. Caregivers identified as a partner $(51.7 \%, n=31)$, parent $(15 \%, n=9)$, child $(13.3 \%, n=8)$, sibling $(5 \%, n=3)$, friend $(3.3 \%, n=2)$, or 'other' $(8.3 \%, n=5)$ with regard to their relationship with the care recipient. The remaining two caregivers (3.3\%) did not specify their relationship to the cancer patient. Caregivers 
identified the cancer type of the care recipient as lymphoma/leukemia $(30 \%, n=18)$, brain $(10 \%, n=6)$, breast $(11.7 \%, n=7)$, colorectal $(11.7 \%, n=7)$, lung $(8.3 \%, n=5)$, and other (e.g., bladder, skin, myeloma; $25 \%$, $n=15)$. The remaining two caregivers (3.3\%) did not specify the type of cancer the care recipient had. Caregivers provided care for one week to up to 264 months, with a median of 12 months, and the number of hours per week of care ranged from about one hour to 168 hours within a week, with a median of 27.5 hours per week.

The mean age of the informal caregiver group was 51.17 (SD of 15.22, range of 19 to 77). Regarding gender, $71.7 \%$ ( $n=43)$ of caregivers identified as female, and the racial/ethnic composition of the caregiver sample included 53 White (88.3\%) four Black (6.7\%), two multiracial (3.3\%), and one Asian $(1.7 \%)$ caregiver. The caregivers were primarily married $(63.3 \%, n=38)$, with others identifying as single $(16.7 \%, n=10)$, divorced $(10 \%, n=6)$, living in a stable relationship $(5 \%, n=3)$, widowed $(3.3 \%, n=2)$, and being separated. $(1.7 \%, n=1)$. Further caregivers reported to have $<\$ 35,000(33.3 \%, n=20)$, had $\$ 35,001$ to $\$ 50,000(18.3 \%, n=11), \$ 50,001$ to $\$ 100,000(26.7 \%, n=16)$, had $>\$ 100,000(18.3 \%, n=11)$ annual income, and two chose not to answer (3.3\%).

The 46 non-caregiver control group participants were recruited using listserv announcements to a university community. The mean age of this group was 36.61 (SD of 12.80, range of 22 to 66). Regarding gender, $95.7 \%(n=44)$ of the control group identified as female, and the racial/ethnic composition of this sample included 45 White (97.8\%) and one multiracial (3.3\%) caregiver. The control group participants were primarily married $(50 \%, n=23)$ with others identifying as single $(32.6 \%, n=15)$, divorced $(6.5 \%, n=3)$, living in a stable relationship $(4.3 \%, n=2)$, widowed $(2.2 \%, n=1)$, and two chose not to answer $(4.3 \%)$. Further, non-caregivers reported to have $<\$ 35,000(17.3 \%, n=8), \$ 35,001$ to $\$ 50,000(13 \%, n=6), \$ 50,001$ to $\$ 100,000(50 \%, n=23),>\$ 100,000(18.3 \%, n=7)$ annual income and two chose not to answer $(4.3 \%)$.

No significant differences between the informal caregiver group and the non-caregiver control group were found for race/ethnicity, $\chi^{2}(3)=4.21, p=.240$, and income, $\chi^{2}(7)=11.84, p=.106$. However, the caregivers were significantly older, $t(104)=5.22, p<.001$, and more likely female, $\chi^{2}(1)=10.18, p=.001$.

\section{Procedure}

The current study is a cross-sectional examination of informal cancer caregiver stress, NAS, depressive symptoms, and salivary cortisol and is part of a larger study (for other relevant publications, see BLINDED). The study was approved by the University of BLINDED Institutional Review Board (IRB NUMBER: 13.0135) and informed consent was obtained from all participants; surveys were completed using an online program or on paper. Data from informal caregivers were collected at the local hospital and during meetings of cancer caregiver support groups. The non-caregiver control group completed the study visit on the university campus.

\section{Measures}


Demographics. Self-reports of gender, weight, height, birthdate, socioeconomic status, and race/ethnicity were collected. Variables relevant for physiological measurement (e.g., allergies, date of last period, gum bleeding, smoking, shift work, time of most recent food, fluid, and other substances) were recorded.

Negative Attribution Style (NAS). To measure the NAS, participants were presented with a hypothetical event (i.e., a caregiving situation for the informal caregivers and a non-caregiving situation for the noncaregivers) and asked to write down one cause for the event. Participants then rate the degree to which the cause of the hypothetical event is (a) internal, (b) stable, and (c) global. Each rating uses a 7-point Likert scale, with higher scores representing a more NAS. Consistent with previous research measuring NAS, the internal consistency in the current study was $a=.60$. This internal consistency is good considering that NAS is measured with only three items. Other instruments measuring NAS show similar internal consistency (36 items, $a=.72 ; 25$ ). Using the Spearman-Brown Formula, the instrument used in our study would have an internal consistency of $a=.94$ if it would have the same length.

Depressive Symptoms. The 20-item Center for Epidemiologic Studies - Depression Scale (CES-D) measures depressive symptoms over the past week [26]. The Cronbach's alpha of the CES-D in a previous caregiver study was $a=.90$ [27], which is consistent with the internal consistency in the current study $(a=.91)$.

Cortisol. Saliva samples were collected between 4:00 PM and 6:00 PM. The passive drooling method was used for collecting a single whole saliva sample over a period of 5 minutes while the participants responded to the survey questions. Saliva was collected in Salivette sampling devices (Sarstedt, Rommelsdorf, Germany) and kept frozen until it was analyzed. Salivary cortisol was measured using the Salivary Cortisol ELISA kit (Salimetrics, Carlsbad, CA). The assay is based on a competitive electrochemiluminescence immunoassay (ELISA) method. The minimal detectable concentration of the method is $0.007 \mathrm{ug} / \mathrm{dL}$. The intra- and inter-assay coefficient of variations are $3-7 \%$ and $3-11 \%$, respectively.

\section{Data Analysis}

Before testing the proposed hypotheses, we z-transformed the NAS scores and calculated the group (cancer caregiver $=1 \mathrm{vs}$. non-caregiver participants $=0$ ) by z-transformed NAS scores interaction scores. Using these scores as predictors, we conducted two linear regression models with depressive symptoms and cortisol as outcome variables, respectively. None of the above listed health variables that have been to contribute to cortisol levels (e.g., allergies, caffeine, date of last period, gum bleeding, hormones, shift work, smoking) in the literature were significant in our sample. Because age and gender were significantly different between informal caregivers and non-caregiver control participants, we included those variables as covariates in both regression models. Control variables were entered in step 1 of the regressions. In step 2, the main effects of group and z-transformed NAS scores were entered as predictors. In step 3, the group by z-transformed NAS scores interaction was entered. To further examine significant group by attribution interaction effects, we constructed model-implied graphs. 


\section{Results}

The descriptive statistics and correlations for the cancer caregiver group and the non-caregiver control participants are presented in Table 1, and the regression results are reported in Table 2 . As expected, the main effects of group $(p=0.001)$ and the group by NAS interaction $(p=0.017)$ significantly predicted depressive symptoms. However, the main effect of NAS was not significant ( $p=0.152)$. Contrary to our hypotheses, the model-implied graph (Figure 1) demonstrated that all caregivers, independent of their NAS, and only non-caregivers high in NAS reported high depression scores. Only non-caregivers low in NAS reported low depression scores. In other words, though non-caregivers low in NAS reported lower depression scores, the other three groups exhibited similar levels of depressive symptoms.

Table 1

Descriptive statistics and correlations for cancer caregivers $(n=60)$ and non-caregiver control participants $(n=43)$

\begin{tabular}{|c|c|c|c|c|c|}
\hline & Attribution & Depressive Symptoms & Cortisol & Mean & SD \\
\hline Attribution & - & .00 & -.19 & 8.81 & 4.35 \\
\hline Depressive Symptoms & $.41^{\star \star}$ & - & -.12 & 18.60 & 10.82 \\
\hline Cortisol & $.27+$ & .22 & - & 0.082 & 0.052 \\
\hline Mean & 11.00 & 14.60 & 0.109 & & \\
\hline SD & 4.21 & 10.72 & 0.069 & & \\
\hline \multicolumn{6}{|c|}{$\begin{array}{l}\text { Note. Data of the cancer caregiver group are presented above the diagonal, data of the non-caregiver } \\
\text { control participants are presented below the diagonal. Attribution = CSQ scores; depressive symptoms } \\
\text { = CES-D scores; Cortisol = cortisol level (ug/dl). }\end{array}$} \\
\hline \multicolumn{6}{|c|}{$+p<.10 ; * p<.05 ; * \star p<.01 ; * \star \star p<.001$} \\
\hline
\end{tabular}


Table 2

Linear regressions with group, attribution style, and their interaction as predictors of depressive symptoms and cortisol

\section{Dependent Variable Depressive Symptoms}

\begin{tabular}{|c|c|c|c|c|c|c|}
\hline & \multicolumn{2}{|c|}{ Step 1} & \multicolumn{2}{|l|}{ Step 2} & \multicolumn{2}{|c|}{ Step 3} \\
\hline & $R^{2}$ & $\beta$ & $R^{2}$ & $\beta$ & $R^{2}$ & $\beta$ \\
\hline Age & & -.121 & & $-.243^{\star}$ & & $-.266^{\star}$ \\
\hline Gender & & .051 & & .063 & & -.057 \\
\hline Group & & & & $.349 * \star$ & & 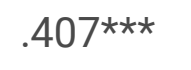 \\
\hline Attribution & & & & $.174+$ & & -.333 \\
\hline Group x Attribution & & & & & & $.569 *$ \\
\hline$R^{2}$ change & .016 & & $.094^{\star \star}$ & & $.011^{\star}$ & \\
\hline Total $R^{2}$ & .016 & & .111 & & .166 & \\
\hline \multirow[t]{3}{*}{ Dependent Variable } & \multicolumn{6}{|c|}{ Cortisol } \\
\hline & \multicolumn{2}{|c|}{ Step 1} & \multicolumn{2}{|l|}{ Step 2} & \multicolumn{2}{|c|}{ Step 3} \\
\hline & $R^{2}$ & $\beta$ & $R^{2}$ & $\beta$ & $R^{2}$ & $\beta$ \\
\hline Age & & $-.210+$ & & -.173 & & -.198 \\
\hline Gender & & -.080 & & -.071 & & -.064 \\
\hline Group & & & & -.040 & & .016 \\
\hline Attribution & & & & .131 & & -.535 \\
\hline Group x Attribution & & & & & & $-.720 \star$ \\
\hline$R^{2}$ change & .055 & & .019 & & $.066^{\star}$ & \\
\hline Total $R^{2}$ & .055 & & .074 & & .140 & \\
\hline \multicolumn{7}{|c|}{$\begin{array}{l}\text { Note. Group = cancer caregiver vs. non-caregiver control participants; Attribution = z-transformed CSC } \\
\text { scores; Depressive Symptoms = CES-D scores; Cortisol = cortisol level (ug/dl). }\end{array}$} \\
\hline$+p<.10 ;{ }^{*} p<.05 ; * \star$ & ${ }^{\star \star} p<$. & & & & & \\
\hline
\end{tabular}

Unexpectedly, the main effects of group $(p=0.920)$ and NAS $(p=0.114)$ did not significantly predict salivary cortisol. However, as hypothesized, the group by NAS interaction $(p=0.036)$ was significantly associated with salivary cortisol levels. Consistent with our hypotheses, the model-implied graph (Figure 2) demonstrated that higher NAS was associated with higher salivary cortisol levels in both groups. 
However, unexpectedly, non-caregivers had higher salivary cortisol levels than caregivers and the impact of NAS on cortisol appears larger in non-caregivers than in informal cancer caregivers.

\section{Discussion}

Following the reformulated helplessness theory [17] and empirical research, we predicted that (a) informal cancer caregiver stress would be associated with elevated levels of depressive symptoms [6, 7] and salivary cortisol (for a review see 8); (b) NAS is positively related to depressive symptoms (for a review see 18) and cortisol independent of cancer caregiver stress; (c) NAS serves as moderator [17] by increasing the effect of informal cancer caregiver stress on depressive symptoms and salivary cortisol. Our results clearly demonstrated that informal caregivers experienced more depressive symptoms than non-caregivers. In addition, NAS was associated with elevated depressive symptoms and cortisol levels in non-caregivers and with cortisol levels in informal caregivers. However, unexpectedly, the cortisol levels in caregivers seem to be lower than in non-caregivers, and NAS seems to be more relevant in non-caregivers than in informal caregivers regardless of the outcome variable (i.e., depressive symptoms, cortisol level).

The fact that we observed effects of NAS on depressive symptoms and cortisol levels in non-caregivers is noteworthy. These findings are consistent with previous literature demonstrating the impact of NAS on psychological (i. e., depressive symptoms; for a review see 18) and physical health [19-23] in the general population. This literature is informative for research examining possible mechanisms by which both psychological and physical health can be improved in the general population.

Our findings that NAS is associated with elevated depressive symptoms and cortisol levels in noncaregivers makes the unexpected finding that informal cancer caregivers report elevated levels of depressive symptoms independent of their NAS even more surprising. This finding might be a reflection of the significant psychological stress causes by informal cancer caregiving. This is in line with the chronicity associated with caregiving in the current sample: an average of 1 year (median: 12 months, range: 1 week to 264 months) for roughly 30 hours each week (median: 27.5 hours per week, range: 1 to 168 hours per week). In other words, the chronic stress associated with informal cancer caregiving overrides any contribution of NAS. To examine this possible explanation a future longitudinal study should attempt to follow informal cancer caregivers from the time of the diagnosis. If our prediction is correct, one would expect that NAS impacts depressive symptoms at the time of the diagnosis and for some time after but that NAS loses its effect with time as caregiving remains a stressor. Thus, a longuditunal study following informal cancer caregivers from the time of the diagnosis on appears to be crucial to elucidate associations among informal cancer caregiving, NAS and depressive symptoms.

One of cortisol's roles is to prepare individuals for the demands of an acute stressor and that, following exposure to an acute stressor, cortisol levels rise to help the body accommodate the increased taxation on the body $[28,29]$. However, in response to chronic stress, cortisol becomes dysregulated [28, 29]. Thus, the severity and chronicity of informal cancer caregiver stress could explain our unexpected finding of higher cortisol levels in non-caregivers than informal cancer caregivers as well as why NAS is associated 
with elevated cortisol levels in both groups but has a stronger impact in non-caregivers than in informal cancer caregivers. Our finding is consistent with some previous studies in which non-caregivers had higher cortisol levels than cancer caregivers [5]. Bevans et al. [5] found that cortisol levels in noncaregivers increased over time, while cortisol levels in cancer caregivers decreased. The authors interpret their results as indication that, over time, cancer caregiver stress wears and tears on the hypothalamicpituitary-adrenal axis to which cortisol belongs. In other words, over time, informal cancer caregiving might be so stressful that not only depressive symptoms are elevated but that the body wears out so even the levels of the stress hormone cortisol are reduced. As described above, a longitudinal study following informal cancer caregivers from the time of the diagnosis appears crucial to test this hypothesis.

\section{Limitations}

Some limitations of our study need be considered. We already highlighted the cross-sectional design as limitation. Thus, the above discussed three-wave longitudinal study design would strengthen the conclusions of our study. An additional limitation is the relative homogeneity of our study sample as the majority of participants identified as female (> 70\%) and White (> $85 \%)$. Given that previous research has identified psychological and physical differences regarding distress depending on gender and race/ethnicity $[7,30]$, authors of future research should take measure to ensure more diverse samples regarding sociodemographic variables. This will broaden the generalizability of findings or allow for the examination of potential moderating effects of gender and race/ethnicity.

The outcomes of our study are limited to depressive symptoms and salivary cortisol. Both are crucial outcomes. Depression is highly prevalent in cancer caregivers (53\%; for a review see 7 ). Cortisol is a commonly used biomarker in measuring the physiological stress response (for a review see 8), which has been associated with physical health issues including CVD [10,11]. Beyond those two outcomes, NAS is related to other physiological measures, like cell-mediated immunity (i.e., T-helper cell/T-suppressor cell ratio, T-lymphocyte response to mitogen challenge; 21) and blood pressure [31]. Based on these additional associations, the exclusive use of only one mental health outcome and one biomarker of physical health could be interpreted as a limitation. Thus, the inclusion of other mental health outcomes and biomarkers should be considered by authors of future studies when evaluating the impact of informal cancer caregiver stress and NAS.

\section{Conclusions}

Informal cancer caregiving is associated with depressive symptoms (for a review see 7) and physiological functioning like elevated levels of cortisol (for a review see 8). Our study demonstrated that while NAS might not impact depressive symptoms in informal caregivers, it is associated with an increase in their cortisol levels. In other words, NAS appears to exacerbate the relationship between informal cancer caregiver stress and cortisol levels. Informal cancer caregivers are crucial members of the treatment team who provide care; however, at the same time, they are also in need of care [32]. By 
being better able to care for themselves, informal caregivers can optimize the care they provide for their loved ones [32], reducing both the likelihood of hospital readmission and related costs for the providing medical facilities [33]. Thus, our findings have relevant implications. Prioritizing interventions, like cognitive behavioral therapy that support informal caregivers in managing their chronic stress and modify their NAS, could positively affect the quality of life for caregivers and their patients.

In sum, despite the limitations of our study, our findings demonstrate effects of informal cancer caregiver stress and NAS on depressive symptoms and salivary cortisol. To expand on our findings, future studies following cancer caregivers from the time of diagnosis using a three-wave longitudinal design and including multiple additional biomarkers of physical health in a racially/ethnically diverse sample appears crucial. If replicated in such studies, our findings demonstrate that the implementation of interventions to support informal caregivers in managing their chronic stress and modify their NAS appears warranted.

\section{Declarations}

\section{Funding}

No funds, grants, or other support was received.

\section{Disclosure of interest}

The authors have no relevant financial or non-financial interests to disclose.

\section{Ethics approval}

All procedures performed in studies involving human participants were in accordance with the ethical standards of the institutional and/or national research committee and with the 1964 Helsinki Declaration and its later amendments or comparable ethical standards. The study was approved by the IRB of the University of BLINDED (No. 13.0135).

\section{Consent to participate}

Informed consent was obtained from all individual participants included in the study.

\section{Data availability statement}

The data that support the findings of this study are available from the corresponding author upon reasonable request.

\section{References}

1. Aoun S (2004) The hardest thing we have ever done: The social impact of caring for terminally ill people in Australia 2004. Deakin West, Palliative Care Australia. 
http://www.palliativecare.org.au/Portals/46/The\%20hardest\%20thing.pdf

2. National Alliance for Caregiving (2016) Cancer caregiving in the U.S.: An intense, episodic, and challenging care experience. Retrieved from http://www.caregiving.org/wpcontent/uploads/2016/06/CancerCaregivingReport_FINAL_June-17-2016.pdf

3. Kim Y, Schulz R (2008) Family caregivers' strain: Comparative analysis of cancer caregiving with dementia, diabetes, and frail elderly caregiving. J Aging Health 20:483-503

4. Jansen L, Dauphin S, Akker M, De Burghgraeve T, Schoenmakers B, Buntinx F (2018) Prevalence and predictors of psychosocial problems in informal caregivers of older cancer survivors-A systematic review: Still major gaps in current research. Eur J Cancer Care 27:1-13

5. Bevans MF, Ross A, Wehrlen L, Klagholz SD, Yang L, Childs R et al (2016) Documenting stress in caregivers of transplantation patients: Initial evidence of HPA dysregulation. Int J Biol Stress 19:175-184

6. Goren A, Gilloteau I, Lees M, daCosta DiBonaventura M (2014) Quantifying the burden of informal caregiving for patients with cancer in Europe. Support Care Cancer 22:1637-1646

7. Girgis A, Lambert S, Johnson C, Waller A, Currow D (2013) Physical, psychosocial, relationship, and economic burden of caring for people with cancer: A review. J Oncol Pract 9:197-202

8. Allen AP, Curran EA, Duggan Á, Cryan JF, Chorcoráin AN, Dinan TG et al (2017) A systematic review of the psychobiological burden of informal caregiving for patients with dementia: Focus on cognitive and biological markers of chronic stress. Neurosci Biobehav Rev 73:123-164

9. Burke HM, Davis MC, Otte C, Mohr DC (2005) Depression and cortisol responses to psychological stress: A meta-analysis. Psychoneuroendocrinology 30:846-856

10. Hamer M, Endrighi R, Venuraju SM, Lahiri A, Steptoe A (2012) Cortisol responses to mental stress and the progression of coronary artery calcification in healthy men and women. PLoS One 7:e31356

11. Hamer M, O’Donnell K, Lahiri A, Steptoe A (2010) Salivary cortisol responses to mental stress are associated with coronary artery calcification in healthy men and women. Eur Heart J 31:424-429

12. Park J, Ross A, Klagholz S, Bevans M (2018) The role of biomarkers in research on caregivers for cancer patients: A scoping review. Biol Res Nurs 20:300-311

13. Corwin EJ, Ferranti EP (2016) Integration of biomarkers to advance precision nursing interventions for family research across the life span. Nurs Outlook 64:292-298

14. Mitchell AM, Pössel P (2017) Repetitive thinking: The link between caregiver burden and depressive symptoms. Oncol Nurs Forum 44:210-216

15. Segerstrom SC, Schipper LJ, Greenberg RN (2018) Caregiving, repetitive thought, and immune response to vaccination in older adults. Brain Behav Immun 22: 744-752

16. Haeffel GJ, Hershenberg R, Goodson JT, Hein S, Square A, Grigorenko EL, et al (2017) The Hopelessness Theory of Depression: Clinical utility and generalizability. Cogn Ther Res 41:543-555

17. Abramson LY, Seligman MEP, Teasdale JD (1978) Learned helplessness in humans: critique and reformulation. J Abnorm Psychol 87:49-74 
18. Liu RT, Kleiman EM, Nestor BA, Cheek SM (2015) The Hopelessness Theory of Depression: A Quarter Century in Review. Clin Psychol 22:345-365

19. Doom JR, Haeffel GJ (2013) Teasing apart the effects of cognition, stress, and depression on health. Am J Health Behav 37:610-619

20. Dykema J, Bergbower K, Peterson C (1995) Pessimistic explanatory style, stress, and illness. J Soc Clin Psychol 14:357-371

21. Kamen-Siegel L, Rodin J, Seligman MEP, Dwyer J (1991) Explanatory style and cell-mediated immunity in elderly men and women. Health Psychol 10:229-235

22. Kubzansky LD, Sparrow D, Vokonas P, Kawachi I (2001) Is the glass half empty or half full? A prospective study of optimism and coronary heart disease in the normative aging study. Psychosom Med 63:910-916

23. Buchanan GM (1995) Explanatory style and coronary heart disease. In Buchanan GM, Seligman MEP (eds) Explanatory Style. Lawrence Erlbaum Associates, Hillsdale, NJ, pp 225-232

24. Mitchell A, Pössel P (2017) Repetitive Negative Thinking: The Link Between Caregiver Burden and Depressive Symptoms. Oncol Nurs Forum, 44:210-216

25. Peterson C, Semmel A, von Baeyer C, Abramson LY, Metalsky GI, Seligman MEP (1982) Cogn Ther Res 6:287-299

26. Radloff LS (1977) The CES-D scale a self-report depression scale for research in the general population. Appl Psychol Meas 1:385-401

27. Carter PA, Chang BL (2000) Sleep and depression in cancer caregivers. Cancer Nurs 23:410-415

28. Chrousos GP, Detera-Wadleigh SD, Karl M (1993) Syndromes of Glucocorticoid Resistance. Ann Intern Med 119:1113

29. Miller GE, Cohen S, Ritchey AK (2002) Chronic psychological stress and the regulation of proinflammatory cytokines: A glucocorticoid-resistance model. Health Psychol 21: 531-541

30. Martin MY, Sanders S, Griffin JM, Oster RA, Ritchie C, Phelan MS, et al (2012) Racial variation in the cancer caregiving experience: A multi-site study of colorectal and lung cancer caregivers. Cancer Nurs 35:249

31. Pössel P, Roane SJ (in press) Depressive Symptoms, Cognitive Styles, and Blood Pressure in Community College Students. J Am Coll Health

32. Harding R, Higginson IJ (2003) What is the best way to help caregivers in cancer and palliative care? A systematic literature review of interventions and their effectiveness. Palliat Med 17:63-74

33. Burke RE, Coleman EA (2013) Interventions to decrease hospital readmissions. JAMA Intern Med 173:695

\section{Figures}




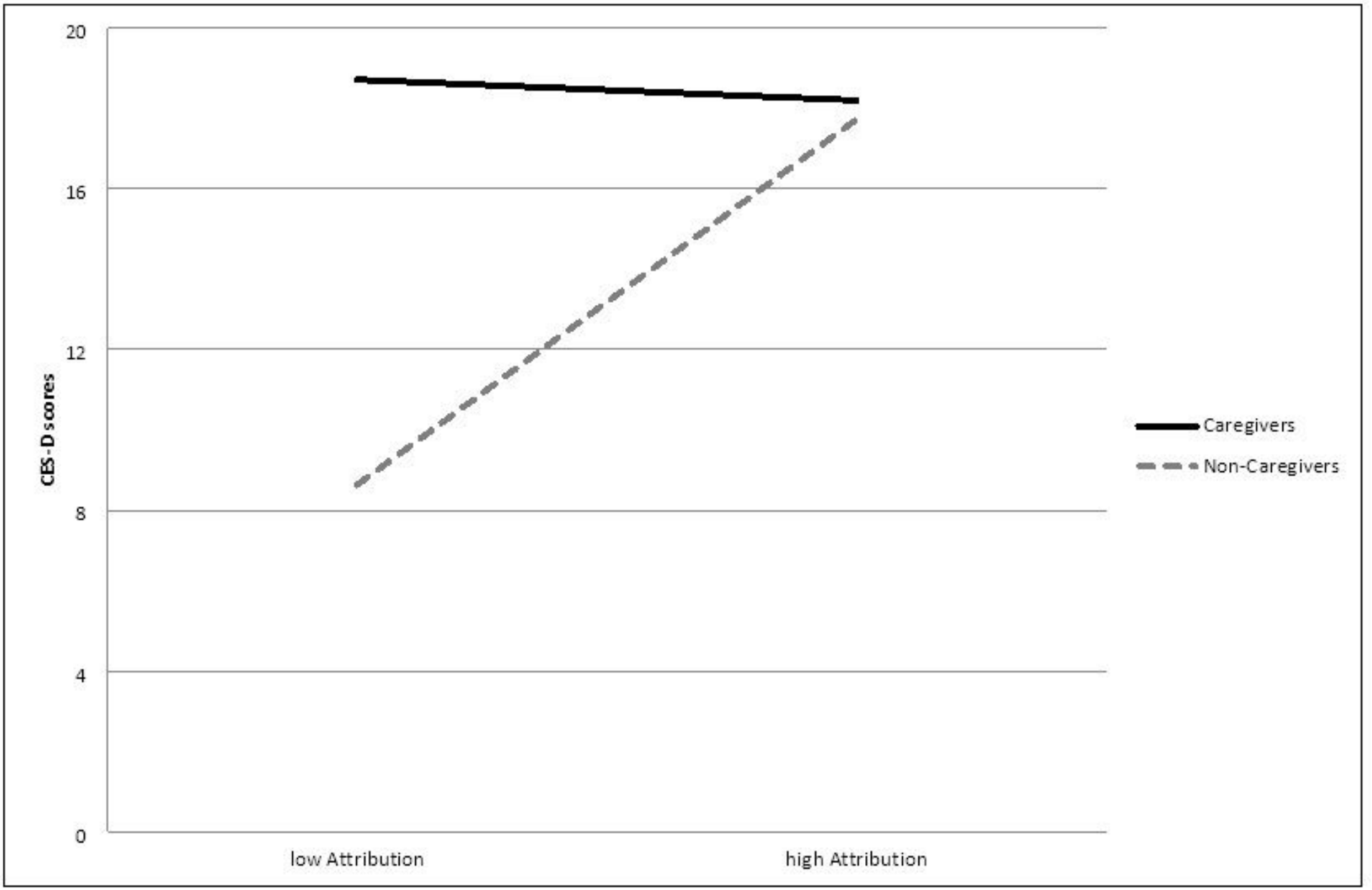

Figure 1

Model-implied graph for the interaction effect of group by negative attribution style on depression scores 


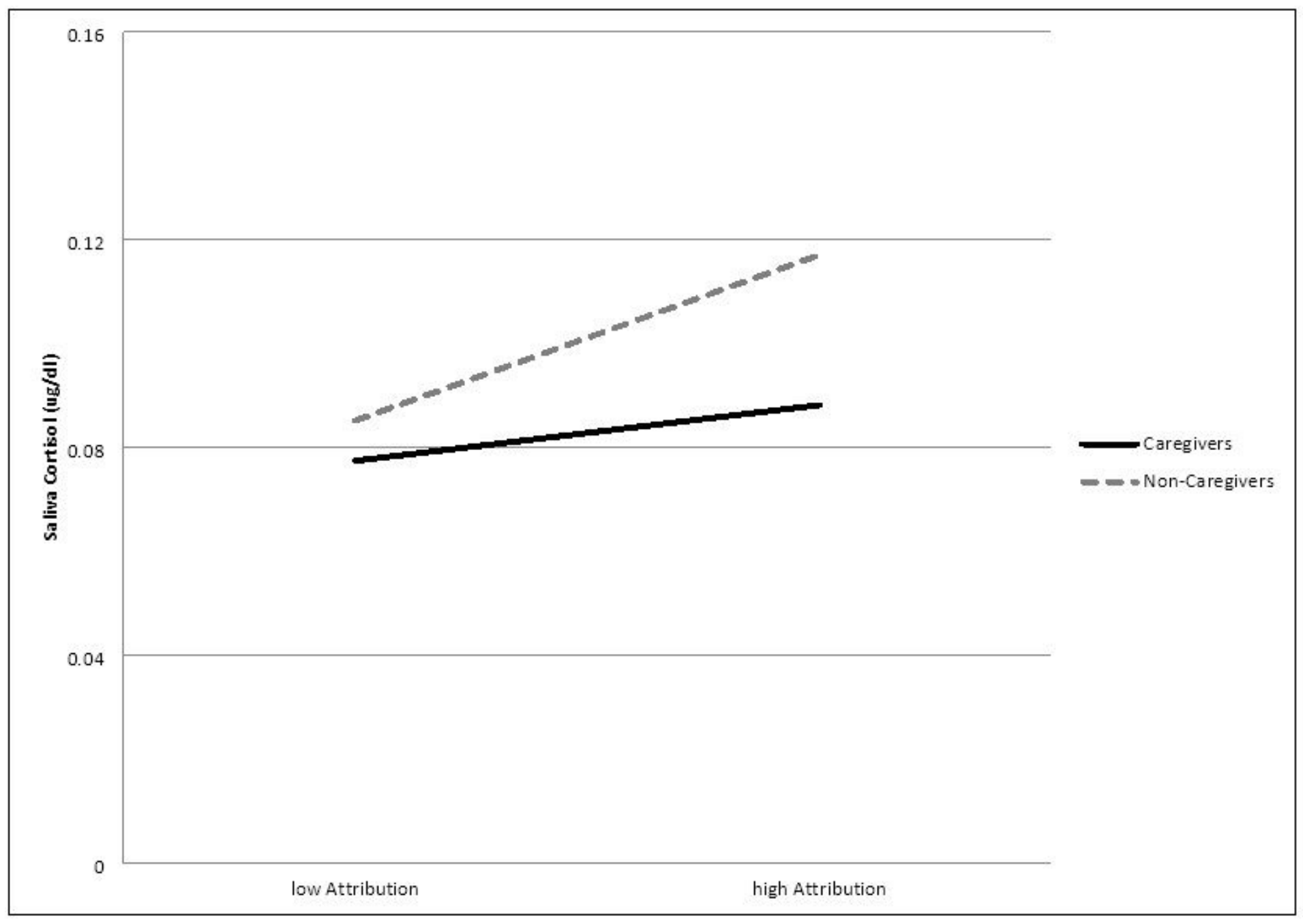

Figure 2

Model-implied graph for the interaction effect of group by negative attribution style on salivary cortisol 